\title{
Lessons from zebrafish on reprogramming the epigenetic code after fertilisation
}

\author{
Chris O’Neill \\ Asian Journal of Andrology (2013) 15, 582-583; doi:10.1038/aja.2013.91; published online 15 July 2013
}

$\mathrm{M}$ ethylation of cytosine bases encodes epigenetic information that governs lineage-specific patterns of gene expression. Remodelling the methylated genome accompanies major transitions during embryo development, including fertilisation, blastulation and gastrulation. The first whole genome epigenetic maps of this modification across these embryonic transitions in a metazoan has been reported in the zebrafish, and shows surprising differences from the long-accepted models developed from more limited analyses of mammalian embryos. The analysis of the zebrafish shows a primacy of the sperm's epigenome as a blueprint for creating the embryonic epigenome. The study illustrates the strengths of comparative approaches to complex biological questions.

Epigenetic information is considered to be encoded within the structure of chromatin. This information provides for lineage specific, mitotically heritable patterns of gene expression that define a cell's structure, function and ontological fate. An understanding of the nature of this epigenetic information and how its lineage specificity is encoded is central to understanding all developmental processes. It is generally thought that normal embryo development requires extensive reprogramming of the epigenetic information inherited from the gametes. One of the most extensively investigated epigenetic signatures is the $5^{\prime}$-methylation of cytosine bases ( $5 \mathrm{meC}$ ) within the genome, particularly at cytosine-guanine $(\mathrm{CpG})$ dinucleotides. Methylation of regulatory regions of the genome is typically associated with their low level of gene expressivity. ${ }^{1}$

Centre for Developmental and Regenerative Medicine, Kolling Institute for Medical Research, Sydney Medical School, University of Sydney, NSW 2065, Australia Correspondence: Dr C O’Neill (chris.oneill@ sydney.edu.au)
Developmental epigenetics in mammals has been dominated for the last couple of decades by a classical model of reprogramming of methyl-CpG within the genome during embryo development. This model holds that fertilisation triggers a process of active global demethylation of the paternallyinherited genome which is followed by a process of passive demethylation of the maternally-derived genome over subsequent mitoses. These processes are considered to 'cleanse' the epigenetic information inherited from the fertilizing gametes (except at imprinted loci) by the end of the preimplantation phase, so that a new embryonic form of epigenetic information can then be imposed upon the genome soon after implantation. ${ }^{2}$

The results of two reports on changes to $5 \mathrm{meC}$ during zebrafish (Danio rerio) embryo development ${ }^{3,4}$ paint a strikingly different picture of reprogramming. The great advantage of these new studies are that they analyses all CpGs within the genome and the methylation levels were distinguished from another important epigenetic mark, 5'-hydroxymethylcytosine. This analysis showed the genome of sperm was more hypermethylated (91\%-average methylation level across the whole genome) than oocytes ( $80 \%)$, and that there were a large number of regions of the genome that were differentially methylated in sperm and oocytes. The zygote and early cleavage stages of development were not assessed but by the 16-cell stage embryos had a level of methylation that approximated the average between the sperm and eggs. The net level of methylation increased as embryos approached the mid-blastula transition (the time of embryonic genome activation), and by the time of gastrulation, the levels of methylation had increased to the level and pattern observed in sperm. The study concludes that at the 16-cell stage both paternally and maternally inherited DNA maintain the methylation patterns inherited from the gametes. Thereafter, there is progressive conversion of differentially methylated regions of the maternally derived genome to resemble the pattern in sperm. Remarkably, the methylation status of the maternally-derived genome was reset to the male pattern as one unit. $^{3}$ Of the large number of loci that were differentially methylated in the sperm and oocyte, most were resolved to the spermspecific pattern by gastrulation. Many of the loci that were differentially methylated encoded genes known to be involved in early embryogenesis, so this provides some reassurance that these reprogramming events are developmentally relevant.

This extensive analysis of reprogramming in the zebrafish shows a profoundly different pattern to the classical model in mammals. This comes at a time when the classical model is undergoing a fundamental re-evaluation. The classical model was built largely on data derived from use of genome-wide analyses by immune localisation of $5 \mathrm{meC}$ or limited and highly selective base-level chemical analyses. Recently, the interpretation of these forms of analyses has been questioned.

The widely reported large loss of $5 \mathrm{meC}$ antigen staining in the paternally-derived pronucleus in the mouse zygote ${ }^{5}$ (which has served as the primary evidence for active global demethylation) was shown to be accounted for by a progressive masking of the antigen during zygotic maturation. ${ }^{6,7}$ This masking was caused by changes in nuclear proteins and could be reversed by brief tryptic digestion of fixed zygotes. When $5 \mathrm{meC}$ staining was performed under conditions favouring antibody binding under thermodynamic equilibrium and after antigen retrieval by a combination of acid-induced denaturation and tryptic digestion of chromatin, $5 \mathrm{meC}$ (and $5 \mathrm{hmC}$ ) were found to persist throughout zygotic maturation and there 
was no evidence of a preferential loss of $5 \mathrm{meC}$ staining from the paternally inherited genome compared to that inherited from the oocyte. ${ }^{6,7}$ In the mouse zygote, $5 \mathrm{meC}$ tends to accumulate at heterochromatic regions and the differing organisation of heterochromatin in the paternally- and maternallyderived genomes, ${ }^{8}$ giving the appearance of differing patterns of methylation.

Base-level analyses of approximately $5 \%$ of the $\mathrm{CpG}$ sites in the mouse zygote (using reduced representation bisulphite sequencing) confirmed that similar levels of methylation exist in the male and female pronuclei, although they were both lower than in sperm. ${ }^{9}$ No global pattern of passive demethylation during subsequent mitoses was evident, but there was some loss of methylation in the cells of the inner cell mass and increased methylation after implantation. While these studies showed that the global patterns of change to methylation in the mouse embryo differed markedly from the predictions of the classical model, they do show that there was evidence of substantial remodelling at many bases. This was particularly evident in $\mathrm{CpG}$ regions associated with LINE and LTR elements of the paternally derived genome, but this was not reported in the zebrafish model. ${ }^{3}$

The interpretation of the functional significance of these base-level $5 \mathrm{meC}$ analyses in the mouse embryo is complicated by the recent discovery that the 'gold standard' methods of chemical analysis of $5 \mathrm{meC}$ (bisulphite conversion and differential restriction analysis) actually provide a net measure of all-known covalent modifications of cytosine $(5 \mathrm{meC}$, $5 \mathrm{hmC}, 5^{\prime}$-formylcytosine and $5^{\prime}$-carboxylcytosine)..$^{10}$ It is also recognized that each of these modifications can provide different epigenetic information to the cell and each have different localisation within the genome. ${ }^{11}$ These limitations have been overcome in the zebrafish analysis by use of modified forms of bisulphite analysis that allow $5 \mathrm{meC}$ to be distinguished from other modifications, ${ }^{3}$ but this is not possible with the small amounts of DNA available from the mammalian embryo. There is much scope for uncertainty about the true pattern of $5 \mathrm{meC}$ reprogramming in the mammalian embryo and much caution is warranted if using the classical model as a hypothetic basis for understanding of developmental epigenetics.

Notwithstanding these limitations in methodology, some information of $5 \mathrm{meC}$ remodelling can be gleaned from the current literature, and this does point to some marked differences between mammals and the zebrafish. There is an overall reduction in the net levels of the cytosine modifications as the mouse zygote develops to the blastocysts stage. ${ }^{9}$ It is likely that this will include loss of $5 \mathrm{meC}$, particularly at repeat elements. By contrast, the level of $5 \mathrm{meC}$ increased over the first embryonic transitions in zebrafish. In the mouse, cytosine modifications undergo remodelling to a pattern broadly similar to the oocyte (although there are many baselevel differences), whereas in the zebrafish, the remodelling is towards the sperm's pattern. Much remodelling occurs at the time of the mid-blastula transition (the time of activation of new transcription from the embryonic genome) in zebrafish, yet in the mouse, this activation occurs at the two-cell stage and there seems to be a relatively modest amount of $5 \mathrm{meC}$ remodelling at this time.

Our understanding of the processes of epigenetic reprogramming of the embryonic genome is clearly in a state of flux. The elegant zebrafish model shows a surprising dominance of the paternally derived genome as a blueprint for the epigenome of the developing embryo, but this is unlikely to be the case in mammals. The finding that such functionally and morphologically divergent structures as the sperm and the gastrulation stage embryo have the same base-level $5 \mathrm{meC}$ patterns raises new questions on the role of this epigenetic mark in lineage specification. The zebrafish will be a powerful model for addressing such questions. Models for reprogramming in mammals are undergoing a major reassessment, yet, the aspects of that model that are reliable do suggest that there are likely to be major divergences in the patterns and processes of epigenetic reprogramming between zebrafish and mammals. If this proves to be the case it may indicate limited evolutionary conservation of this process, a finding entirely consistent with a current view that these processes are highly plastic and provide a major source of phenotypic variation within populations. Further detailed comparative analyses of model species across phyla will provide powerful new insights into developmental epigenetics.

\section{COMPETING FINANCIAL INTERESTS}

The author declares that there are no competing financial interests.

1 Klose RJ, Bird AP. Genomic DNA methylation: the mark and its mediators. Trends Biochem Sci 2006 31: 89-97.

2 Morgan HD, Santos F, Green K, Dean W, Reik W. Epigenetic reprogramming in mammals. Hum $\mathrm{Mol}$ Genet 2005; 14: R47-58.

3 Jiang L, Zhang J, Wang J, Wang L, Zhang L et al. Sperm, but not oocyte, DNA methylome is inherited by zebrafish early embryos. Cell 2013; 153: 773-84.

4 Potok M, Nix D, Parnell T, Cairns B. Reprogramming the maternal zebrafish genome after fertilization to match the paternal methylation pattern. Cell 2013; 153: 759-72.

5 Mayer W, Niveleau A, Walter J, Fundele R, Haaf T. Demethylation of the zygotic paternal genome. Nature 2000; 403: 501-2.

6 Li Y, O'Neill C. Persistence of cytosine methylation of DNA following fertilisation in the mouse. PLOS ONE 2012; 7: e30687.

7 Li Y, O'Neill C. 5'-methylcytosine and 5'-hydroxymethylcytosine each provide epigenetic information to the mouse zygote. PLOS ONE 2013; 8: e63689.

8 Salvaing J, Aguirre-Lavin T, Boulesteix C, Lehmann G, Debey $\mathrm{P}$ et al. 5-methylcytosine and 5-hydroxymethylcytosine spatiotemporal profiles in the mouse zygote. PLOS ONE 2012; 7: e38156.

9 Smith ZD, Chan MM, Mikkelsen TS, Gu H, Gnirke A et al. A unique regulatory phase of DNA methylation in the early mammalian embryo. Nature 2012; 484: 339-44.

10 Huang Y, Pastor WA, Shen Y, Tahiliani M, Liu DR et al. The behaviour of 5-hydroxymethylcytosine in bisulfite sequencing. PLOS ONE 2010; 5: e8888.

11 Yu M, Hon G, Szulwach K, Song CX, Zhang L et al. Base-resolution analysis of 5-hydroxymethylcytosine in the mammalian genome. Cell 2012; 149: 136880 